\title{
Waist-to-chest ratio predicts the reward value of men's bodies linearly, but not quadratically
}

Benedict Jones ${ }^{1}$ (ben.jones@glasgow.ac.uk), Danielle Morrison ${ }^{1}$, Amanda Hahn $^{2}$, Lisa DeBruine

1. Institute of Neuroscience \& Psychology, University of Glasgow

2. Department of Psychology, Humboldt State University

Sell et al. [1] found that cues of upper body strength were a particularly good predictor of women's judgments of men's physical attractiveness. As they noted, some theories of men's physical attractiveness suggest that particularly masculine-, strong-looking men will be relatively unattractive. In other words, these theories predict a quadratic, rather than linear, effect of strength cues on men's physical attractiveness. Consequently, it is striking that Sell et al. [1] found a strong positive linear effect of strength cues on women's ratings of men's physical attractiveness, but observed no evidence for a quadratic effect of these strength cues.

In a recent paper [2], we measured women's responses to images of men's bodies using a standard key-press task that is commonly used to measure stimulus reward value and in which longer viewing times indicate more rewarding images. In light of Sell et al's [1] results, we used multilevel modeling to reanalyse these [2] data and test whether viewing times were linearly predicted by waist-to-chest ratio (an important cue of men's upperbody strength and formidability, [3]) and also whether there was a quadratic relationship between viewing times and waist-to-chest ratio.

Our analysis suggested that women found men's bodies with more masculine waist-to-chest ratios more rewarding (i.e., there was a significant linear effect of waist-to-chest ratio, $t=-2.48, p=.016)$, but showed no evidence of a quadratic effect of waist-chest ratio on the reward value of men's bodies $(t=0.21, p=0.83)$. These results are consistent with Sell et al's [1] conclusion that women find strong, masculine male bodies optimally attractive and do not show the aversions to highly masculine bodies that some theories might predict. We suggest that investigating the extent to which women's responses to men's strength cues vary as a function of ecological and cultural factors could provide further insights into the function and development of these preferences.

Full results and code for these analyses are publicly available on the Open Science Framework at https://osf.io/s9b3n/. The body stimuli used in this study are publicly available on the Open Science Framework at https://osf.io/2j9qd/.

1. Sell, A., Lukazsweski, A. W. \& Townsley, M. (2017). Cues of upper body strength account for most of the variance in men's bodily attractiveness. Proc. R. Soc. B, 284, 20171819. DOI: 10.1098/rspb.2017.1819 
2. Morrison, D., Wang, H., Hahn, A. C., Jones, B. C. \& DeBruine, L. M. (2017). Predicting the reward value of faces and bodies from social perception. PLoS ONE 12, e0185093. DOI: 10.1371/journal.pone.0185093

3. Fan, J., Dai, W., Liu, F. \& Wu, J. (2005). Visual perception of male body attractiveness. Proc. R. Soc. B, 272, 219-226. DOI: 10.1098/rspb.2004.2922 